\title{
Quantitative Evaluation of Petal Shape and Picotee Color Pattern in Lisianthus by Image Analysis
}

\author{
Yosuke Yoshioka and Ryo Ohsawa \\ Graduate School of Life and Environmental Sciences, University of Tsukuba, 1-1-1 Tennodai, Tsukuba, \\ Ibaraki 305-8572, Japan \\ Hiroyoshi Iwata and Seishi Ninomiya ${ }^{1}$ \\ Department of Information Science and Technology, National Agricultural Research Center, 3-1-1 \\ Kannondai, Tsukuba, Ibaraki 305-8666, Japan \\ Naoko Fukuta
Department of Genetics and Physiology, National Institute of Floricultural Science, 2-1 Fujimoto,
Tsukuba, Ibaraki 305-8519, Japan
}

ADDITIONAL INDEX WORDS. elliptic Fourier descriptor, Eustoma grandiflorum, principal component analysis, comparative marginal distribution

\begin{abstract}
Petal shape and picotee colour pattern of lisianthus [E ustoma grandiflorum (Raf.) Shinn.] were qualitatively evaluated by means of personal computer-based methods. In lisianthus, many cultivars have been improved to obtain various floral characteristics. Picotee color patterns and flower shape are commercially important in this species and the availability of an objective and quantitative evaluation method is of vital importance for investigations related to the genetic and physiological aspects of these characteristics. Our objectives were to evaluate petal shape variation quantitatively and to establish a new quantitative evaluation method for picotee color patterns. We succeeded in quantitatively evaluating petal shape variation by means of elliptic Fourier descriptors and principal-components analysis, and in evaluating picotee color patterns by a newly developed procedure based on comparative marginal distribution. Petal shape variation was divided into symmetrical and asymmetrical elements of the entire shape variation. Both groups were additionally divided into several components. The variations in picotee color pattern were effectively described by the first through fourth principal components. Comparing the varietal effect of these components, nested analyses of variance showed that the differences between cultivars in picotee color pattern were smaller than those of the symmetrical shape elements. In addition, the environmental effects on picotee color formation were greater than those of symmetrical shape formation. The evaluation methods described in this study are effective for further investigations, and are applicable to other floricultural crops as well as lisianthus.
\end{abstract}

Floral characteristics such as flower shape and color pattern are important targets for improvement of floriculture crops because these characteristics are directly related to the commercial value of the products. In lisianthus, the floral characteristics have long been genetically improved to meet different consumer demands. Among various floral characteristics improved, picotee color formation has been of interest commercially. This species originated in the United States, and its commercial cultivars have been intensively developed in Japan (Halevy and Kofranek, 1984). A cultivar with purple picotee color was first commercially available in $1986, \approx 50$ years after introduction of this species to Japan. In subsequent years, breeders and breeding companies have bred some dozens of picotee color cultivars, most of which are $\mathrm{F}_{1}$ hybrids. Today, an important target of breeding in lisianthus is stability or uniformity within a cultivar in phenotypic expression of floral characteristics, because genetic heterogeneity exists within a cultivar and the phenotypic expression is affected by cultural conditions, especially in picotee color formation (Fukuta et al., 2005). Concurrently, development of an adequate cultivation method to increase the yield of high-quality cut flowers is expected.

In this context, many researchers and breeding companies have carried out various studies to obtain an appropriate understanding

Received for publication 5 July 2005. Accepted for publication 23 Nov. 2005. We thank Dr. Masanori Honjo of the Univ. of Tokyo for critical reading of the manuscript.

'To whom reprint requests should be addressed; E-mail: snino@affrc.go.jp of the genetic and developmental mechanisms that govern floral characteristics. Although picotee color has been quantified by a colorimeter, other floral characteristics, such as petal shape and picotee color pattern, have been evaluated qualitatively (Fukuta and Nakayama, 2003), even if quantitative variations in such characteristics were observed. Qualitative evaluation by human visual judgment, which is often used, sometimes results in unacceptable human errors, and training and experience are required for accurate and consistent assessment. In addition, qualitative evaluation of floral characteristics is definitely inadequate to evaluate continuous variation due to genotypes and cultural conditions. Therefore, an objective and quantitative evaluation method is vital, not only for lisianthus, but for other floricultural crops as well.

Digital image analysis is one way to compensate for the weakness of the current qualitative evaluation of continuous variation in various characteristics. With recent improvements in computer performance, digital image analysis has been applied to quantitative evaluation in various agricultural and biological research, such as disease assessment (Martin and Rybicki, 1998; Niemira et al., 1999; Olmstead and Lang, 2001), measurement of various plant canopies (Adamsen et al., 1999; Ewing and Horton, 1999; Karcher and Richardson, 2003; Lukina et al., 1999; Olmstead et al., 2004; Purcell, 2000; Richardson et al., 2001), and morphological analysis (Iwata et al., 1998; Iwata and Ukai, 2002; Theobald et al., 2004; Yoshioka et al., 2004a, 2004b, 2005). In the evaluation of petal shape, a combination of elliptic Fourier descriptors and 
principal-components analysis (EF-PCA) seemed to be most effective among several methods suggested. EF-PCA describes overall petal shape mathematically by transforming coordinate information concerning its contour into elliptic Fourier coefficients (Kuhl and Giardina, 1982), and summarizes these coefficients by means of principal-components analysis (Rohlf and Archie, 1984). Meanwhile, there are few proposed methods to evaluate flower color pattern. Yoshioka et al. (2004b) suggested a simple method to evaluate the flower color pattern of Primula sieboldii E. Morren. However, this method is not adequate for the picotee color pattern of lisianthus, because it evaluates only the central part of the overall petal, and neglects information about other parts. Therefore, the development of an appropriate method for picotee color pattern evaluation is highly desirable.

In this study, we describe image-based methods to quantitatively evaluate petal shape and picotee color pattern in lisianthus. We first evaluated petal shape variation by means of EF-PCA, and developed a quantitative evaluation method for picotee color patterns by PCA of the data derived from comparative marginal distribution (CMD). We then evaluated the varietal contribution to the petal shape and picotee color pattern by means of nested analysis of variance (ANOVA) of the scores for each principal component (PC).

\section{Materials and Methods}

Plant Materials. There are $\approx 25$ to 30 commercial cultivars of lisianthus that have picotee petals in Japan. We randomly chose $15 \mathrm{~F}_{1}$ hybrid and true-bred cultivars regardless of petal shape and picotee color pattern (numbers in parentheses are cultivar identification numbers): Candy Marine (1), Neo Swallow (2), Mira Marine 4 (3), Kyo no Warabe (4), Clear Marine (5), Candy Dolphin (6), Neo Marine (7) Spica Marine 18 (8), Kyono Suzu (9), Asuka no Nami (10), Excel Marine (11), Maike Sky (12), Excel Navy Ring (13), San Sarf 26 (14), and Moret Marine (15) (Fig. 1). Genetic heterogeneity among each cultivar exists because lisianthus is an allogamous plant. The plants were grown in a greenhouse at the National Institute of Floricultural Science (Ibaraki, Japan) from Feb. to July 2004. We randomly sampled five plants per cultivar and three flowers per plant. We used all the petals of each flower (15 cultivars $\times 5$ plants $\times 3$ flowers $\times 5$ petals $=1125$ petals).

IMAGE RECORDING. Before scanning, we separated the five petals from each flower with a regular kitchen knife. We obtained petal images using a digital image scanner (GT-9800F; Epson Co., Tokyo), which is suitable for obtaining plain petal images (Yoshioka et al., 2004b). We placed 15 petals (from one plant) simultaneously on the flat bed of the scanner. Since petals of most cultivars had almost two-dimensional structure, the influences of flatting these petals by flat bed cover were considered to be negligible. We fixed a sheet of red paper in the flat bed cover so that the contrast between the flat bed cover and the samples became clear, and scanned the samples at 300 dots/inch resolution. We saved each image in red-green-blue (RGB) color format (BMP) with 256 levels (i.e., 8-bit resolution per channel).

Evaluation OF PETAl SHAPE USING EF-PCA.After we converted each petal image into a binary image using a threshold method, we obtained the closed contour of the petal and chain-coded it (Freeman, 1974) using programs developed using JBuilder X (Borland Software Corp., Cupertino, Calif.). The coefficients of the elliptic Fourier descriptors (EFDs), which were normalized to avoid variations related to the size, rotation, and starting point of

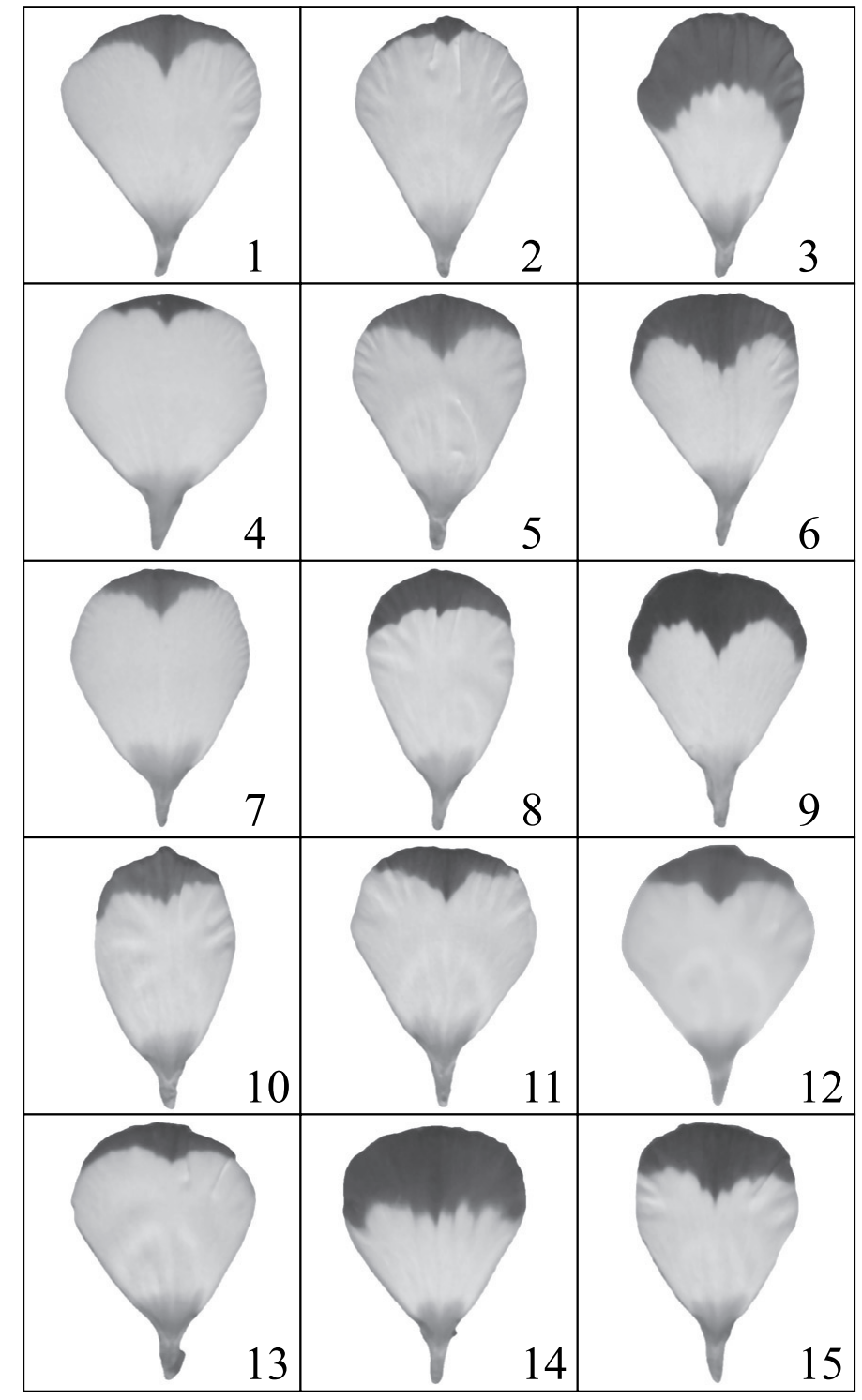

Fig. 1. Photographs of petals from cultivars of lisianthus used in this study.

the contour trace, were then calculated from the chain-code data using the procedure proposed by Kuhl and Giardina (1982). By this procedure, we approximated the shape of each petal using the first 20 harmonics, and thus calculated $80(4 \times 20)$ standardized EFDs. The 80 coefficients could be classified into two groups related to symmetrical variations and asymmetrical variations (Iwata et al., 1998; Yoshioka et al., 2004a). We refer to the former group as the symmetrical group and to the latter as the asymmetrical group. To summarize the information contained in the coefficients in each group, we performed a PCA based on a variance-covariance matrix. To determine the effect of each PC on petal shape, we recalculated the coefficients of the EFDs, letting the score on a particular PC equal the mean $\pm 2 \mathrm{SD}$, while keeping the scores of the remaining components as means. A series of above processes (EF-PCA), without the chain-coding, was carried out using the program SHAPE developed by Iwata and Ukai (2002). We used the scores of the PCs as the characteristics of petal shape in the statistical analyses.

QuAntitative evaluation OF PICOTEe COlOR PATTERN. We calculated the total petal area [PA (square centimeters)] based on the number of pixels occupied by the petal image. We classified 
each pixel into a picotee or non-picotee category according to its RGB values, and used this information to create binary petal images. The ranges of RGB values of picotee area were determined for the classification in a pilot study. We then defined the ratio of the picotee area to the PA as the number of picotee pixels divided by the total pixel area of the petal, and used this ratio as an index of the picotee color proportion (PCP). Each petal image was rotated around a center of gravity so that the central axis of the petal corresponded approximately to a vertical line, and was resized so that the petal width corresponded to 200 pixels. That is, each petal image became an aggregate of 200 vertical lines, each one pixel wide. From each modified image, we created a comparative marginal distribution (CMD; Fig. 2): we calculated the 200 variables that represent the ratio of pigmentation pixels to petal pixels on each vertical line.

To summarize the CMD data, we performed a PCA based on the variance-covariance matrix by R 1.7.1 (R Development Core Team, 2005). In order to determine the effect of each PC on the picotee color pattern, we reconstructed picotee color patterns using the 200 variables calculated in reverse by the same method as petal shape, and redrew petal images by applying recalculated CMD data to average petal shape, which was calculated by EFPCA. The evaluation of picotee color pattern was carried out using programs developed using JBuilder X. We used the scores of the PCs as the characteristics of picotee color pattern in the statistical analyses.

Statistical Analysis. To examine the varietal effect on each PC score relative to variations in petal shape and picotee color pattern, we performed nested ANOVA because the samples had a hierarchical structure: cultivar, plant, flower, and petal. Nested ANOVA was performed using the following linear model:

$\omega_{i j k l}=\mu+\alpha_{i}+\beta_{i j}+\gamma_{i j k}+\varepsilon_{i j k l}$

where $\omega_{i j k l}$ denotes the score of each principal component of $i$ th cultivar, the $j$ th plant, the $k$ th flower and the $l$ th petal. The $\mu$ is the grand mean, $\alpha_{i}$ is the effect of $i$ th cultivar, $\beta_{i j}$ is the effect of $j$ th plant nested within $i$ th cultivar, $\gamma_{i j k}$ is the effect of $k$ th flower nested within $j$ th plant of $i$ th cultivar, and $\varepsilon_{i j k l}$ is the random experimental error. In this nested ANOVA, we estimated each significant level by F test, and calculated the proportion of the variance component in each hierarchical source. Then we regarded the proportion due to the difference between cultivars as varietal effect. We also analyzed PA and PCP in a similar fashion. In addition, Pearson's product-moment correlation analyses were also performed in order to investigate the relationships between petal shape and picotee color pattern. Correlation analyses used the mean values for each plant and flower. All statistical analyses were performed using JMP (version 4.0; SAS Institute, 2000).

\section{Results}

Agood summary of the standardized EFDs was provided by the first four PCs of the symmetrical group and of the asymmetrical group. The symmetrical group had a larger component of overall shape variation than did the asymmetrical group (Table 1). Figure 3 shows the effect of the PCs of both groups on petal shape. These reconstructed shapes indicate that the first and second PCs of the symmetrical group are good measures of the aspect ratio and the position of the center of gravity, accounting for $67.7 \%$ and $9.2 \%$ of the overall petal shape variation, respectively (Fig. 3, Table 1). The third and fourth PCs of the symmetrical group are associated with petal curvature. Meanwhile, the PCs of the asymmetrical

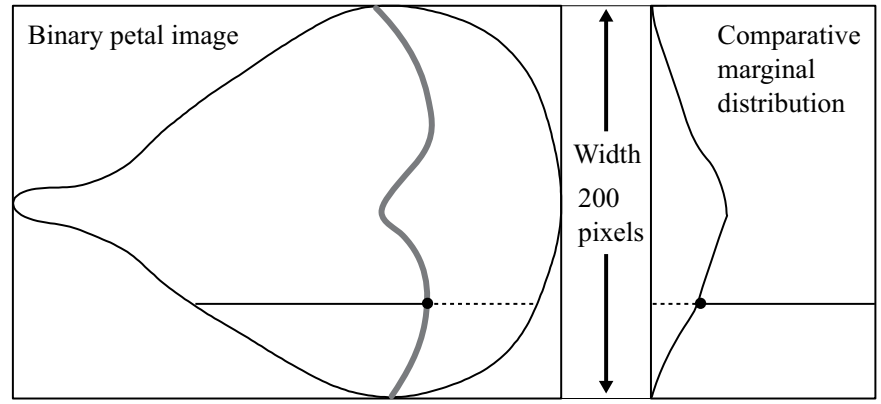

Fig. 2. Schematic diagram of the comparative marginal distribution (CMD: right histogram) made from binary petal image (left petal illustration) of lisianthus. In the left petal illustration, a thick curved line represents a boundary between picotee and non-picotee area. The CMD data of each petal consists of 200 variables that represent the ratio of pigmentation pixels to petal pixels on each horizontal line that could be drawn on the scan of each petal.

group express the horizontal skewness from the mean petal shape (Fig. 3). That is, the first to the fourth PCs of the asymmetrical group express the differences between the left and right parts of the bottom, sides, and top of the petal, respectively.

The eigenvalues and the contributions of the first four PCs of the picotee color pattern are shown in Table 1. The contribution of the first PC was very high and accounted for as much as $87.4 \%$ of the total variation. The cumulative contribution from the first to the fourth $\mathrm{PC}$ is $96.6 \%$. Figure 4 shows the reconstructed image of each PC. These images indicate that the first PC is a good measure of the ratio of pigmentation area in the total petal. In fact, this PC was highly correlated with PCP $(r=-0.985, N$ $=1200, P<0.001)$. Therefore, we excluded the PCP from the subsequent analysis. The second and third PCs express the patterns of pigmentation area, accounting for $6.5 \%$ and $1.6 \%$ of the total picotee pattern variation. The fourth PC seems to affect the difference in pigmentation area between the right and left parts of the petal.

Significant differences were observed in all characteristics among the 15 cultivars (Table 2). Scatterplots clearly indicate the wide variations in petal characteristics (Figs. 5 and 6). From these results, we were able to characterize the cultivars. For example, in the petal shape variation of the symmetrical group, 'Asuka no Nami' (10) and 'Spica Marine' (8) have the lowest first and second PC scores because of their narrow petals and low centers of gravity (Figs. 1 and 5). The petals of 'Candy Marine' (1), in contrast, are the widest and have a high center of gravity (Figs. 1 and 5). In the picotee color pattern, the petals of 'Neo Swallow' (2) and 'Neo Marine' (7), which have small picotee areas, show high scores in the first PC of CMD (Figs. 1 and 6). In contrast, 'Mira Marine 4' (3) and 'San Sarf 26' (14) show low scores in the first PC of CMD, indicating that the petals of these cultivars have large pigmentation areas (Figs. 1 and 6). 'Mira Marine 4' (3) shows the lowest score in the second PC and the highest score in the third PC. The specific values of 'Mira Marine 4' (3) are evident in Fig. 1.

The proportion of the variance component due to the varietal effect in the nested ANOVA was higher than that for any other components (plant, flower, and petal) in PA and the PCs of the symmetrical group (Table 2). In contrast, the largest proportion of the variance component was due to the differences between petals in each PC of the asymmetrical group and the fourth PC of CMD. For the remaining characteristics, the largest proportions of the variance component were due to the differences between plants in the first and second PCs of CMD, and between flow- 
Table 1. Contributions of principal components in symmetrical and asymmetrical groups of elliptic Fourier descriptors (EFDs) related to petal shape variation of lisianthus, and those in comparative marginal distribution (CMD) data related to variation of picotee color patterns of lisianthus. EFDs of each petal were calculated mathematically by transforming coordinate information concerning its contours. Each group of EFDs consists of 40 coefficients. CMD data of each petal consists of 200 variables that represent the ratio of pigmentation pixels to petal pixels on each vertical line that could be drawn on the scan of its petal.

\begin{tabular}{|c|c|c|c|c|c|}
\hline \multirow[b]{3}{*}{ Component } & \multirow{2}{*}{\multicolumn{2}{|c|}{$\frac{\text { Symmetrical group (shape) }}{\text { Proportion }(\%)}$}} & \multirow{2}{*}{\multicolumn{2}{|c|}{$\frac{\text { Asymmetrical group (shape) }}{\text { Proportion }(\%)}$}} & \multirow{3}{*}{$\frac{\text { CMD data }}{\text { Proportion }(\%)}$} \\
\hline & & & & & \\
\hline & of group ${ }^{z}$ & of totaly & of group ${ }^{z}$ & of totaly & \\
\hline 1 & 78.5 & 67.7 & 41.8 & 8.0 & 87.4 \\
\hline 2 & 10.7 & 9.2 & 28.0 & 5.4 & 6.5 \\
\hline 3 & 4.0 & 3.5 & 11.4 & 2.2 & 1.6 \\
\hline 4 & 2.7 & 2.3 & 5.6 & 1.1 & 1.1 \\
\hline
\end{tabular}

Proportion relative to the total variance of the elliptic Fourier coefficients belonging to each group.

yProportion relative to the total variance of all coefficients of both groups. also confirm the effectiveness of EF-PCA in the evaluation of lisianthus petals. The variations in petal shape in lisianthus can be partitioned into symmetrical and asymmetrical features. The greatest variation in petal shape is explained by the symmetrical feature, because it accounted for a larger proportion of the total variation (Table 1). The PCs in each group were independent of each other, and thus can be used as new shape characteristics of lisianthus. In addition, except for the aspect ratio (the first PC of the symmetrical group), the shape variations described by the PCs of both groups were previously difficult to measure quantitatively.

This is the first time that picotee color pattern has been quantitatively evaluated. Our newly developed procedure has three

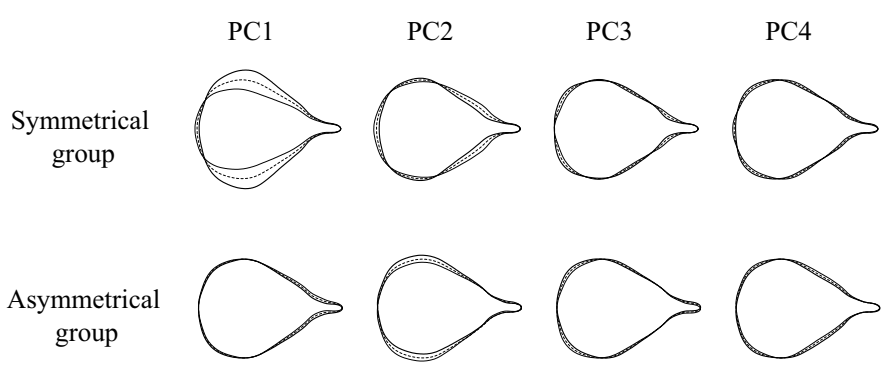

Fig. 3. Effect of each principal component in symmetrical and asymmetrical groups of elliptic Fourier descriptors (EFDs) on petal shape of lisianthus. EFDs of each petal were calculated mathematically by transforming coordinate information concerning its contours. Each group of EFDs consists of 40 coefficients. Each shape was reconstructed from coefficients calculated by letting the score for the corresponding principal component be equal to its mean or its mean plus or minus two times the SD, and setting the scores on the remaining components at zero. Dashed line, thick solid line, and thin solid line stand for mean, mean $+2 \mathrm{SD}$, and mean $-2 \mathrm{SD}$.

ers in the third PC of CMD. In correlation analyses using plant and flower means, we did not observe any strong relationships between petal shape and picotee color pattern.

\section{Discussion}

The visual impression of flowers directly influences consumer preference and commercial values. Petals are the main visual component of flowers, and thus directly or indirectly affect such appetites and values. Petal shape and picotee color pattern were previously measured as qualitative characteristics because of difficulties in measuring them. In this study, we successfully measured the quantitative differences between cultivars and between plants within a cultivar in lisianthus. Our quantitative measurements are more appropriate for investigating these characteristics than are the commonly used qualitative measurements. We evaluated the two-dimensional shapes and picotee color patterns of petals using several independent components, which are thought to be major morphological factors affecting the impressions given by flowers. Our approach could be used to investigate floral characteristics in many other horticultural crops.

In previous studies, EF-PCA showed remarkable results in the evaluation of several plant organs (Furuta et al., 1995; Iwata et al., 1998, 2002; Ohsawa et al., 1998; Yoshioka et al., 2004a). We can major advantages. First, this procedure can accurately detect small quantitative variations in picotee color pattern. Although the second, third, and fourth PCs accounted for $6.5 \%, 1.6 \%$, and $1.1 \%$ of the total variance, respectively (Table 1 ), the varietal differences of these PCs were highly significant. These components are difficult to score by human visual assessment. Second, this procedure can evaluate the picotee color pattern independent of size and shape. In correlation analyses, PCs of the picotee color pattern were not correlated with PAs and PCs of symmetrical and asymmetrical petal shapes. This independence is a great advantage because human visual assessment is often deceived and misled by size and shape factors. Third, we could visually

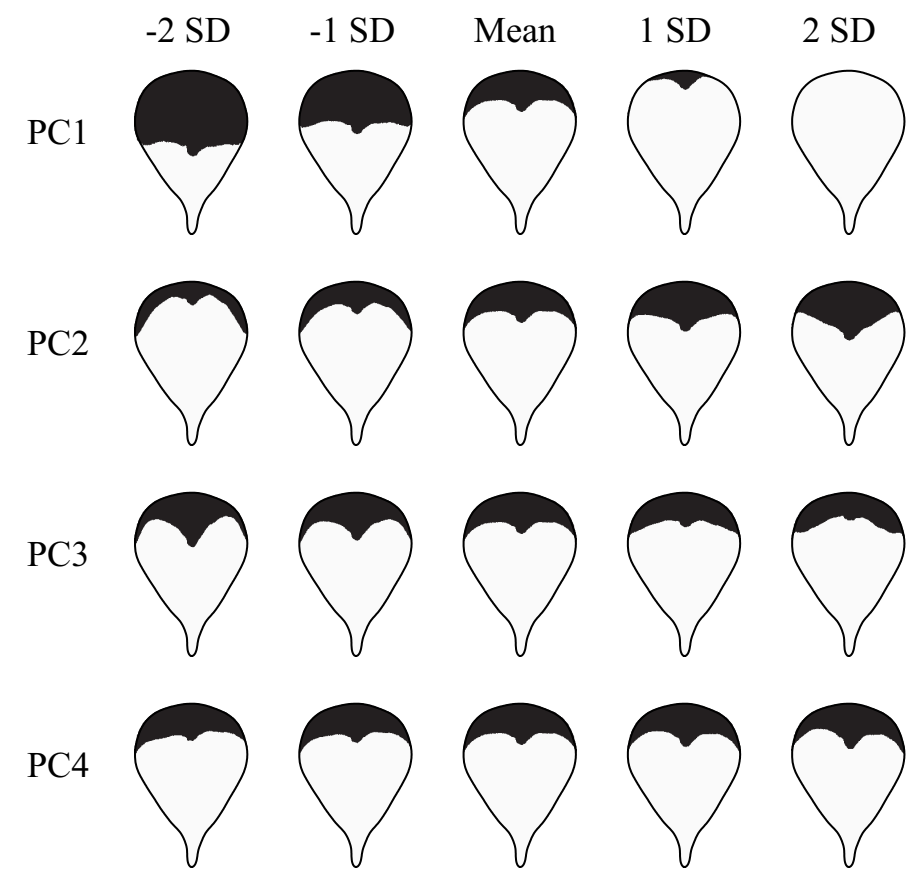

Fig. 4. Effect of each principal component in the comparative marginal distribution (CMD) data on picotee color pattern of lisianthus. The CMD data consists of 200 variables that represent the ratio of pigmentation pixels to petal pixels on each horizontal line that could be drawn on the scan of each petal. Each petal illustration was redrawn from CMD data calculated by the score for the corresponding principal component be equal to its mean, mean plus or minus the SD, and setting the scores on the remaining components at zero. From the right, each column shows the case where the score takes $+2 \mathrm{SD},+\mathrm{SD}$, mean, $-\mathrm{SD}$, and $-2 \mathrm{SD}$, respectively. 
Table 2. Proportions (\%) of variance components in petal area (PA) and the principal components (PC) of elliptic Fourier descriptors (EFDs) related to the petal shape (symmetrical and asymmetrical groups) and picotee color pattern (comparative marginal distribution data: CMD) of lisianthus derived from each of the four hierarchical sources: cultivar, plant, flower, and petal (as estimated by nested ANOVA). EFDs of each petal were calculated mathematically by transforming coordinate information concerning its contours. Each group of EFDs consists of 40 coefficients. CMD data of each petal consists of 200 variables that represent the ratio of pigmentation pixels to petal pixels on each vertical line that could be drawn on the scan of its petal.

\begin{tabular}{|c|c|c|c|c|c|}
\hline \multirow[b]{2}{*}{ Source } & \multicolumn{5}{|c|}{ Principal components of group A (shape) } \\
\hline & PA & PC1 & PC2 & PC3 & PC4 \\
\hline Cultivar & $54.7^{* *}$ & $67.5^{* *}$ & $54.7^{* *}$ & $64.0^{* *}$ & $39.6^{* *}$ \\
\hline Plant (cultivar) & $4.7 \mathrm{Ns}$ & $15.0^{* *}$ & $7.2^{* *}$ & $0.0^{\mathrm{NS}}$ & $7.0^{* *}$ \\
\hline Flower (cultivar, plant) & $37.7^{* * *}$ & $5.6^{* *}$ & $8.3^{* *}$ & $16.3^{* *}$ & $25.5^{* *}$ \\
\hline \multirow[t]{2}{*}{ Petal (cultivar, plant, flower) } & 3.0 & 11.9 & 29.8 & 19.8 & 28.0 \\
\hline & \multicolumn{4}{|c|}{ Principal components of group B (shape) } & \\
\hline source & PC1 & PC2 & PC3 & PC4 & \\
\hline Cultivar & $9.1^{* *}$ & $11.1^{\text {*** }}$ & $31.1^{* *}$ & $42.6^{* *}$ & \\
\hline Plant (cultivar) & $3.7^{*}$ & $5.2^{* *}$ & $4.5^{* *}$ & $5.3^{* *}$ & \\
\hline Flower (cultivar, plant) & $3.3^{\mathrm{NS}}$ & $2.0^{\mathrm{NS}}$ & $1.3^{\mathrm{NS}}$ & $6.9^{* *}$ & \\
\hline \multirow[t]{2}{*}{ Petal (cultivar, plant, flower) } & 84.0 & 81.6 & 63.1 & 45.2 & \\
\hline & \multicolumn{4}{|c|}{$\begin{array}{c}\text { Principal components of the } \\
\text { histogram data (picotee color patten) }\end{array}$} & \\
\hline Source & PC1 & $\mathrm{PC} 2$ & PC3 & $\mathrm{PC} 4$ & \\
\hline Cultivar & $41.7^{* *}$ & $33.0^{* *}$ & $9.0^{*}$ & $22.6^{* *}$ & \\
\hline Plant (cultivar) & $54.0^{* *}$ & $46.7^{* *}$ & $25.8^{* *}$ & $10.5^{* *}$ & \\
\hline Flower (cultivar, plant) & $3.3^{* *}$ & $13.6^{* *}$ & $45.1^{* *}$ & $14.7^{* *}$ & \\
\hline Petal (cultivar, plant, flower) & 1.0 & 6.7 & 20.1 & 52.1 & \\
\hline
\end{tabular}

NS, ***Nonsignificant or significant at $P<0.05$ or 0.01 , respectively.
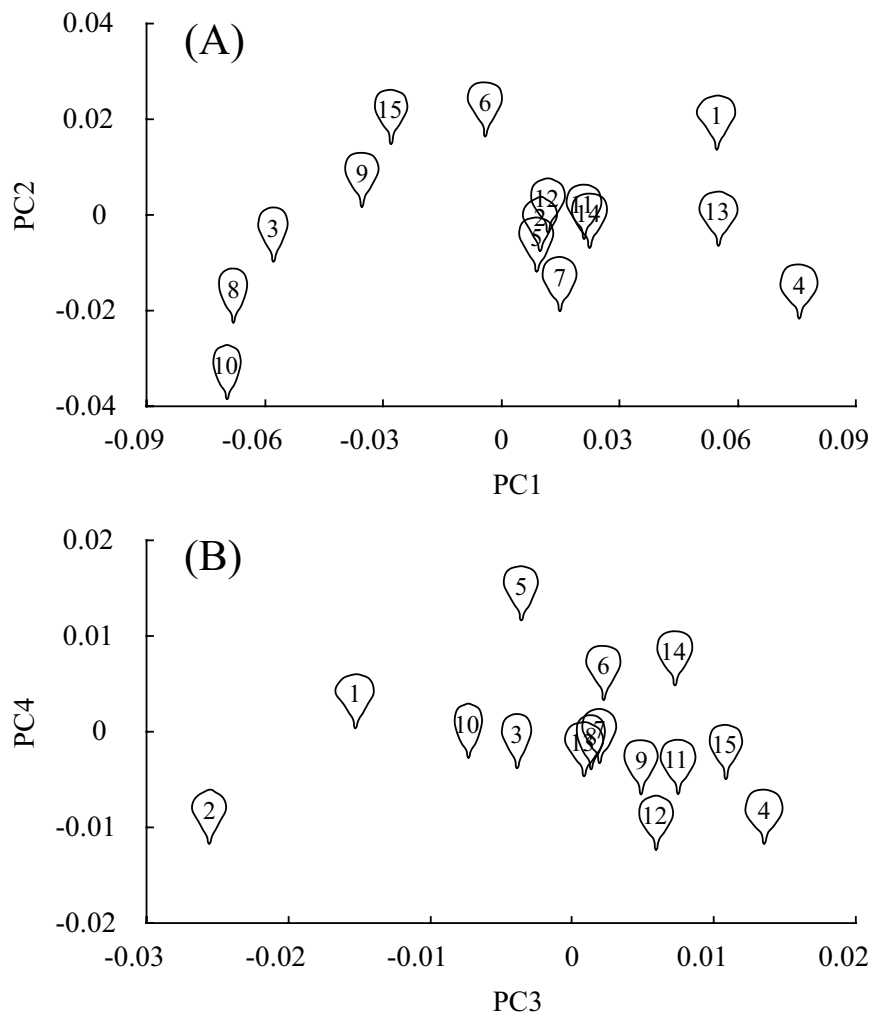

Fig. 5. Petal shape variations in the 15 cultivars of lisianthus. (A) the first vs .second principal component (PC) of symmetrical group; (B) the third vs. fourth PC of symmetrical group. The symbols for the 15 cultivars examined in this study represent their mean petal shapes. Numbers correspond to the numbers of the cultivars described in the Materials and Methods. observe how each PC affects the picotee color pattern by drawing the patterns under particular scoring conditions (Fig. 4). This visualization, combined with the other two advantages, is very effective for performing further analyses related to the genetic and physiological aspects of picotee color pattern.

The variations in picotee color pattern in lisianthus are mainly composed of four characteristics (PC scores of CMD data): the ratio of pigmentation area to nonpigmentation area in the total petal, the pattern of the pigmentation area, and the differences in pigmentation area in the right and left parts of the petal (Fig. 4). These characteristics can be classified into symmetrical and asymmetrical features. That is, the first to third PCs of CMD data belong to the symmetrical category, and the fourth PC of CMD data belongs to the asymmetrical category. The symmetrical category accounts for most of the variations in picotee color pattern, because the first three PCs of CMD data account for most of the total variation (Table 1).

Although not designed to estimate heritability, our significant varietal effects in the nested ANOVAs indicate that there are genetic components to the petal characteristics examined in this study. However, environmental factors observed in the plant and flower hierarchies are also important. These results indicate that the variations in petal shape and picotee color pattern among plants within a cultivar, and among flowers within a plant, are brought about by the external environment around each plant, or by developmental instability within a plant or flower.

Previous studies (Yoshioka et al., 2004a, 2004b) concluded that it is appropriate to consider the asymmetrical variation in petal shape and color pattern as being strongly affected by environment rather than by genotype. However, this appears to not be the case for lisianthus petals, indicating that the genetic or developmental mechanisms in the asymmetrical feature vary by the asymmetrical characteristic. Although we could observe large proportions due to the differences between petals in the first and second PCs of group B and the fourth PC of CMD data, the varietal effects in the third and fourth PCs are relatively large (Table 2). In addition, tendencies to have unidirectional asymmetrical petals were observed in several cultivars. For example, most of the petals in 'Candy Marine' (1) were rightward-deformed. These results indicate that the asymmetrical variations in the bottom and side parts of the petal shape and in picotee color pattern have arisen from environmental effects. In contrast, the latter asymmetrical features (PC3: asymmetrical variation in the top part of petal, PC4: the effect cannot be clearly understood) would be strongly controlled by genotype.

Fukuta and Nakayama (2003) reported that formation of the picotee color pattern is subject to environmental effects, especially temperature change. In this study, we reared all plants under the same cultural conditions, which is thought to be most suitable for developing stable picotee color patterns in cut flowers (Fukuta et al., 2005). Nonetheless, differences in picotee color formation were observed between plants and between flowers within a cultivar. As we expected, the variance components are largest in the differences between plants for the first and second PCs of 

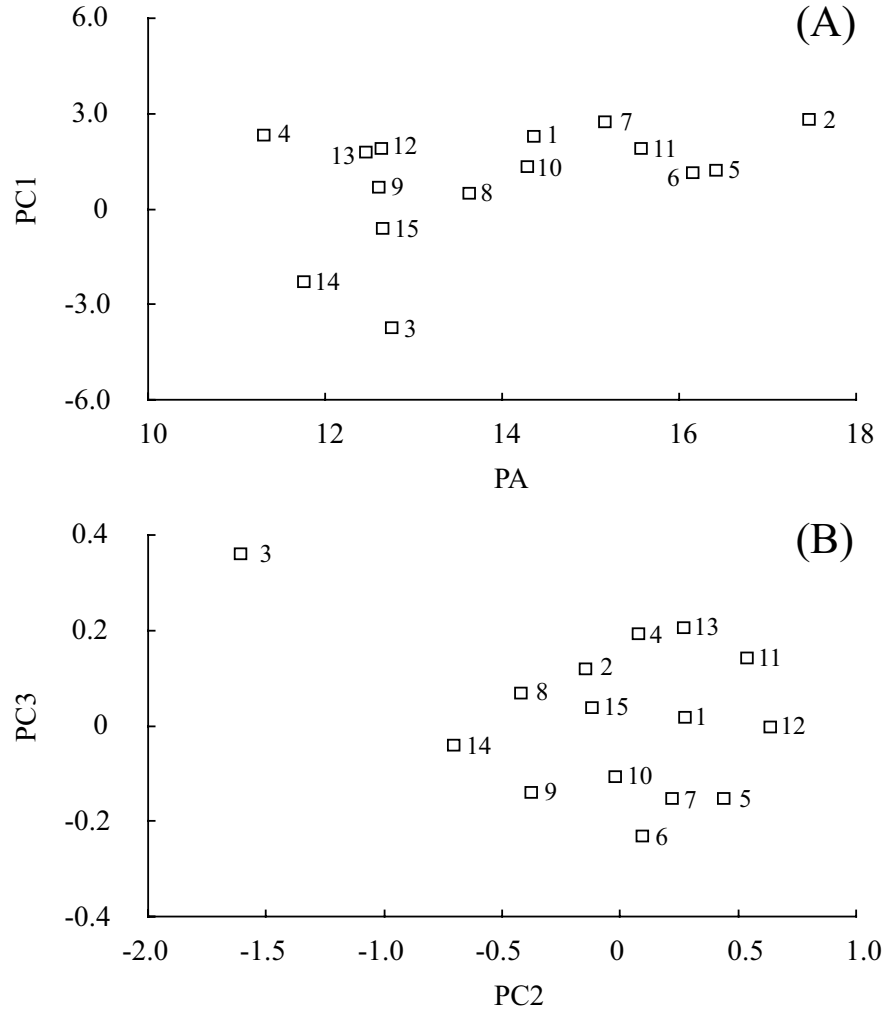

Fig. 6. Variation of petal area and picotee color pattern in the 15 cultivars of lisianthus. (A) petal area (PA) vs. first principal component (PC) of the comparative marginal distribution (CMD) data; (B) second vs. third $\mathrm{PC}$ of the CMD data. The square symbols indicate the means of the cultivars. Numbers correspond to the numbers of cultivars described in Materials and Methods.

CMD data, and between flowers for the third PC (Table 2). These results indicated that we can now quantitatively evaluate the instability of picotee color pattern formation with much greater accuracy than was previously possible. In addition, the differences between cultivars in the symmetrical formation of picotee color pattern are less than those in the symmetrical shape formation, because the varietal effects of picotee color pattern are smaller than those of the symmetrical shape variations (Table 2). Therefore, the stability or uniformity of picotee color pattern within a cultivar is likely to be less than that of petal shape.

In conclusion, we have described computer-aided procedures to evaluate variations in petal shape and picotee color pattern; these procedures could also reveal some relationships between these petal characteristics and genetic and environmental factors. These procedures require only an easily accessible commercial computer and a digital scanner. Our results suggest that these procedures can be used efficiently to evaluate petal characteristics of lisianthus and other plants in scientific research and breeding projects.

\section{Literature Cited}

Adamsen, F.J., P.J. Pinter, Jr., E.M. Barnes, R.L. LaMorte, G.W. Wall, S.W. Leavitt, and B.A. Kimball. 1999. Measuring wheat senescence with a digital camera. Crop Sci. 39:719-724.

Ewing, R.P. and R. Horton. 1999. Quantitative color image analysis of agronomic images. Agron. J. 91:148-153.

Freeman, H. 1974. Computer processing of line drawing images. Assn. Computing Machinery Computing Surveys 6:57-97.
Fukuta, N. and M. Nakayama. 2003. The effect of low temperature on picotee formation in Eustoma grandiflorum (in Japanese). J. Jpn. Soc. Hort. Sci. 72 (2):471 (Abstr.).

Fukuta, N., R. Ohsawa, Y. Yoshioka, and M. Nakayama. 2005. Quantitative evaluation of the inter-varietal variation in picotee formation stability of Eustoma grandiflorum (in Japanese). Hort. Res. 4:265-269.

Furuta, N., S. Ninomiya, S. Takahashi, H. Ohmori, and Y. Ukai. 1995. Quantitative evaluation of soybean (Glycinemax L., Merr.) leaflet shape by principal component scores based on elliptic Fourier descriptor. Breeding Sci. 45:315-320.

Halevy, A.H.,and A.M. Kofranek. 1984. Evaluation of lisianthus as a new crop. HortScience 19:845-847.

Iwata, H., H. Niikura, S. Matsuura, Y. Takano, and Y. Ukai. 1998. Evaluation of variation of root shape of Japanese radish (Raphanus sativus L.) based on image analysis using elliptic Fourier descriptor. Euphytica 102:143-149.

Iwata, H. and Y. Ukai. 2002. Shape: A computer program package for quantitative evaluation of biological shapes based on elliptic Fourier descriptors. J. Hered. 93:384-385.

Karcher, D.E. and M.D. Richardson. 2003. Quantifying turfgrass color using digital image analysis. Crop Sci. 43:943-951.

Kuhl, F.P. and C.R. Giardina. 1982. Elliptic Fourier features of a closed contour. Computer Graphics Image Processing 18:236-258.

Lukina, E.V., M.L. Stone, and W.R. Raun. 1999. Estimating vegetation coverage in wheat using digital images. J. Plant Nutr. 22:341-350.

Martin, D.P. and E.P. Rybicki. 1998. Microcomputer-based quantification of maize streak virus symptoms in Zea mays. Phytopathology 88:422-427.

Niemira, B.A., W.W. Kirk, and J.M. Stein. 1999. Screening for late blight susceptibility in potato tubers by digital analysis of cut tuber surfaces. Plant Dis. 83:469-473.

Ohsawa, R., T. Tsutsumi, H. Uehara, H. Namai, and S. Ninomiya. 1998. Quantitative evaluation of common buckwheat (Fagopyrum esculentum Moench) kernel shape by elliptic Fourier descriptor. Euphytica 101:175-183.

Olmstead, J.W. and G.A. Lang. 2001. Assessment of severity of powdery mildew infection of sweet cherry leaves by digital image analysis. HortScience 36:107-111.

Olmstead, M.A., R. Wample, S. Greene, and J. Tarara. 2004. Nondestructive measurement of vegetative cover using digital image analysis. HortScience 39:55-59.

Purcell, L.C. 2000. Soybean canopy coverage and light interception measurements using digital imagery. Crop Sci. 40:834-837.

R Development Core Team. 2005. R: A language and environment for statistical computing. R Foundation for Statistical Computing, Vienna, Austria. 27 Sept. 2005. <http://www.r-project.org>.

Richardson, M.D., D.E. Karcher, and L.C. Purcell. 2001. Quantifying turfgrass cover using digital image analysis. Crop Sci. 41:1884-1888.

Rohlf, F.J. and J.W. Archie. 1984. A comparison of Fourier methods for the description of wing shape in mosquitoes (Ritera culicidae). Systematic Zoology 33:322-317.

SAS Institute. 2000. JMP statistics and graphics guide, version 4. SAS Inst., Cary, N.C.

Theobald, C.M., C.A. Glasbey, G.W. Horgan, and C.D. Robinson. 2004. Principal component analysis of landmarks from reversible images. Appl. Stat. 53:163-175.

Yoshioka, Y., H. Iwata, R. Ohsawa, and S. Ninomiya. 2004a. Analysis of petal shape variation of Primula sieboldii E. Morren by elliptic Fourier descriptors and principal component analysis. Ann. Bot. 94:657-664.

Yoshioka, Y., H. Iwata, R. Ohsawa, and S. Ninomiya. 2004b. Quantitative evaluation of flower colour pattern by image analysis and principal component analysis of Primula sieboldii E. Morren. Euphytica 139:179-186.

Yoshioka Y., H. Iwata, R. Ohsawa, and S. Ninomiya. 2005. Quantitative evaluation of the petal shape variation in Primula sieboldii caused by breeding process in the last 300 years. Heredity 94:657-663. 